\title{
A Comparison of Validated Methods Used to Assess Impaired Awareness of Hypoglycaemia in Type 1 Diabetes: An Observational Study
}

\author{
Kshitiz Ghandi · Beatrice Pieri · Anne Dornhorst · Sufyan Hussain (D)
}

Received: September 24, 2020 / Accepted: October 30, 2020 / Published online: November 20, 2020

(c) The Author(s) 2020

\begin{abstract}
Introduction: Clarke, Gold and Pedersen are validated methods to assess awareness of hypoglycaemia. Identifying impaired awareness of hypoglycaemia (IAH) is critical for supporting people with structured education and diabetes technologies, to reduce harm of hypoglycaemia. This study compares the Clarke score, Gold score and Pedersen methods and their correlations with features of hypoglycaemia unawareness and patient characteristics, to evaluate the accuracy of the methods in identifying IAH.

Methods: This retrospective, observational questionnaire-based study collected routine clinical data from 100 people with type 1 diabetes. The questionnaire included the three
\end{abstract}

Electronic Supplementary Material The online version of this article (https://doi.org/10.1007/s13300020-00965-0) contains supplementary material, which is available to authorized users.

K. Ghandi · A. Dornhorst · S. Hussain ( $\square)$ Department of Diabetes, Endocrinology and Metabolism, Imperial College London, London, UK e-mail: Sufyan.hussain@kcl.ac.uk

K. Ghandi · A. Dornhorst · S. Hussain Department of Diabetes and Endocrinology, Imperial College Healthcare NHS Trust, London, UK

B. Pieri · S. Hussain

Department of Diabetes, School of Life Course

Sciences, King's College London, London, UK validated scoring methods, frequency of severe and nocturnal hypoglycaemia, knowledge and worry of hypoglycaemia and hypoglycaemia symptom scores using the Edinburgh Hypoglycaemia Scale. Data were analysed for IAH prevalence and the associations with features of IAH. The concordance of Clarke, Gold and Pedersen methods was evaluated using Spearman's correlation coefficient.

Results: The prevalence of IAH in this cohort identified by Clarke, Gold and Pedersen methods was $18 \%, 19 \%$ and $61 \%$ respectively. The mean autonomic symptom score in people with IAH was significantly reduced using the Clarke method $(P=0.0002)$ but not on Gold $(P=0.12)$ and Pedersen methods $(P=0.79)$. For people with IAH assessed using the Clarke method, scores for night-time worry regarding hypoglycaemia $(P=0.04)$ and self-reported frequency of nocturnal hypoglycaemia $(P=0.001)$ were increased. Spearman's correlation coefficients between Pedersen and Clarke and Pedersen and Gold were $R_{\mathrm{s}}=0.555(P<0.001)$ and $R_{\mathrm{s}}=0.645$ $(P<0.001)$ respectively. A moderate association was observed between Clarke and Gold $R_{\mathrm{s}}=$ $0.5669(P<0.001)$.

Conclusion: Whilst Clarke and Gold methods determined a similar prevalence of IAH, people identified with IAH assessed by the Clarke method had a significant association with the features and characteristics of IAH, including reduced autonomic symptoms. This study suggests that performing more than one score is 
important for a reliable risk assessment of IAH.

Keywords: Hypoglycaemia; Hypoglycaemia unawareness; Impaired awareness of hypoglycaemia; Type 1 diabetes

\section{Key Summary Points}

Recurrent hypoglycaemia remains an important cause for morbidity and mortality in type 1 diabetes. Impaired awareness (IAH) of hypoglycaemia is common and results in inappropriate behavioural and physiological responses to restore blood glucose leading to recurrent hypoglycaemia.

Recognition and assessment of IAH is critical to direct appropriate medical, behavioural and educational treatment. Current strategies to diagnose IAH in clinical settings rely on the use of validated methods based on patient questionnaires.

This observational study aims to compare three validated methods used to assess impaired awareness of hypoglycaemia in a large city teaching hospital.

This study reveals differences between characteristics of people with IAH defined by Clarke, Gold and Pedersen methods.

The study highlights that care must be taken when using these methods in clinical and research settings given their non-equivalence and that multiple methods of assessment should be considered to obtain a reliable risk assessment of IAH.

\section{DIGITAL FEATURES}

This article is published with digital features, including a summary slide, to facilitate understanding of the article. To view digital features for this article go to https://doi.org/10. 6084/m9.figshare.13154327.

\section{INTRODUCTION}

Despite advances in diabetes management, hypoglycaemia still remains a major adverse effect of insulin treatment and barrier to achieve optimal glycaemic control in diabetes [1]. Hypoglycaemia accounts for significant morbidity, mortality, worry and impacts on quality-of-life, employment and ability to drive in people with type 1 diabetes mellitus [2-7].

The widespread use of intensified insulin treatment and lower glycaemic targets in the management of type 1 diabetes mellitus may promote the development of impaired awareness of hypoglycaemia (IAH) [8]. IAH results in a marked reduction in hypoglycaemic symptoms and is also associated with attenuated counterregulatory hormonal responses to hypoglycaemia [8]. Therefore, IAH results in inappropriate behavioural and physiological responses to restore blood glucose that leave patients exposed to the effects of hypoglycaemia more frequently and for a longer duration [9]. Furthermore, increased recent and recurrent exposure to hypoglycaemia leads to further impairment of awareness [10].

Recognition and assessment of IAH is critical to direct appropriate medical, behavioural and educational treatment [11]. Structured education, such as DAFNE (Dose Adjustment for Normal Eating), and new diabetes technologies, such as CGM (continuous glucose monitoring) and CSII (continuous subcutaneous insulin infusion), are used to reduce to reduce episodes of severe hypoglycaemia and restore awareness of hypoglycaemia [11-14]. Assessment of IAH is also important for meeting current European driving standards [7]. Current strategies to assess IAH in clinical settings rely on the use of validated methods based on patient questionnaires. Three validated methods have been used extensively in this regard $[9,15,16]$. This study aims to compare three validated methods used to assess impaired awareness of hypoglycaemia in a large city teaching hospital. The prevalence 
of IAH and its associations with features of IAH and patient characteristics were investigated. This retrospective study also illustrated the difficulty in recognising IAH in clinical settings. The aims of this study are to compare the validated questionnaire-based methods for assessing IAH with clinical characteristics and clinical features of hypoglycaemia unawareness and therefore to provide an assessment of accuracy for these methods in assessing IAH.

\section{METHODS}

\section{Study Population}

One hundred adults with type 1 diabetes mellitus attending an urban adult diabetes outpatient clinic between January 2016 and August 2016 undertook a routine clinic questionnaire detailed below. Exclusion criteria consisted of pregnancy, advance renal failure and inability to understand or complete the questionnaire. Four adults were unable to complete the questionnaire. Two were excluded because of language difficulties and a further two were excluded because of incomplete responses. A member of the clinical team was present to assist with clarification of the content of the questionnaire if needed. Baseline demographic and clinical information was assessed using patient records. Haemoglobin A1c was measured using a standard method on the Menarini HA-8160 (Menarini Pharma, UK) analyser. The results were Diabetes Control and Complications Trial (DCCT) aligned; the local reference range for $\mathrm{HbA} 1 \mathrm{c}$ is $5.0-6.5 \%$.

The study was not required to have institutional review board (IRB) approval as it used existing routine clinical data accessed directly by the clinical team caring for the patients and met local requirements to be undertaken as a clinical audit. No intervention was implemented on the patients for the purpose of the study, and no patient-identifiable information was used in the study. Retrospective analysis of questionnaires used in routine practice was approved as an audit by Imperial College Healthcare NHS Trust Clinical governance office. Informed consent was obtained from all participants at the time of questionnaire completion.

\section{Questionnaire and Assessment of IAH}

A single questionnaire incorporated the validated hypoglycaemia awareness scoring methods of Gold et al., Clarke et al. and PedersenBjergaard et al. $[9,15,16]$. The questionnaire included the question 'how often in the last month have you had readings $<3.5 \mathrm{mmol} / \mathrm{L}$ at night?' with responses being 'Never', ' 1 to 3 times/month','1 time/week', '4.5 times/week' and 'almost every night', to assess frequency of nocturnal hypoglycaemia. Hypoglycaemia symptom scores were assessed using the Edinburgh Hypoglycaemia Scale in this questionnaire [17]. The retrospective recall of severe hypoglycaemia over a period of 1 year, which is a robust measure in people with type 1 diabetes was also assessed in this questionnaire [18]. A seven-point Likert scale with 1 representing 'never' or 'strongly disagree' and 7 representing 'always' or 'strongly agree' was used to assess knowledge to avoid future hypoglycaemic episodes, daytime and night-time worry for hypoglycaemia. IAH and normal awareness (NA) was assessed as per the scoring methods provided by Clarke et al., Gold et al. and Pedersen et al. $[9,15,16]$. On the Gold and Clarke methods, a score of 4 or more implied IAH $[9,15]$. Using the Pedersen method people who answered 'usually' or 'never' were considered to have IAH [16]. Self-reported poor symptoms of hypoglycaemia were also assessed separately as a cause for hypoglycaemia using a seven-point Likert scale with 1 representing 'strongly disagree' and 7 representing 'strongly agree'.

\section{Statistical Analysis}

All analyses were performed using SPSS version 14.0 for Windows (SPSS Inc., Chicago, IL, USA). A $P$ value less than 0.05 was considered to be significant. Initial comparisons between hypoglycaemic aware and unaware groups for continuous variables were performed using the twosample Student's $t$ test and those for non-continuous variables were performed using a 
Mann-Whitney $U$ test. For categorical variables, differences in proportions between the groups were compared using the chi-square test or Fisher's exact test when necessary. To assess the relationship between two variables Spearman's correlation, Matthews' correlation coefficient and kappa score were used. All results are reported as mean \pm SEM (standard error of mean) unless indicated. Results as mean \pm SD (standard deviation) are provided in the supplementary material.

\section{RESULTS}

\section{Baseline Characteristics}

The questionnaire was completed by 100 adults (46 male) with type 1 diabetes (Table 1 ). Mean \pm SEM for age (years) $44 \pm 1.4$; duration of diabetes $21.8 \pm 1.4$ years; glycated haemoglobin (HbA1c) $69.6 \pm 2 \mathrm{mmol} / \mathrm{mol}$; body mass index (BMI) in men $25.7 \pm 0.6 \mathrm{~kg} / \mathrm{m}^{2}$; BMI in women $26.0 \pm 0.7 \mathrm{~kg} / \mathrm{m}^{2}$. Among the cohort, $78 \%$ were using insulin analogues, $19 \%$ a mixture of short-acting analogue and long-acting human insulin and 3\% were using human insulins alone. $83 \%$ used a basal-bolus regimen, $11 \%$ used CSII and 3\% were on twice or thrice daily regimens of fixed insulin mixtures; $51 \%$ of people had completed a diabetes structured education programme.

\section{Prevalence and Patient Characteristics of IAH}

The prevalence of IAH as identified by the Clarke, Gold and Pedersen methods was $18 \%$, $19 \%$ and $61 \%$ respectively (Table 1 ). The prevalence of IAH was $7 \%$ if a revised version of the Pedersen method was used where "always and usually" represented normal awareness and "occasionally and never" represented IAH.

People with IAH had a significantly longer duration of diabetes $(P=0.002,0.02$ and 0.04 on Clarke, Gold and Pedersen methods respectively), increased age on Clarke method only $(P=0.002)$ as compared to normal awareness. There were no significant differences in insulin regimen and insulin type between both groups. There was a significantly increased number of severe hypoglycaemic episodes in the previous year for people with IAH using the Clarke $(P=0.02)$ and Pedersen $(P=0.04)$ methods but not the Gold method.

\section{Hypoglycaemia Symptom Scores}

The mean autonomic symptom score in people with IAH as compared to normal awareness was significantly reduced using the Clarke method $(P=0.0002)$ but not on Gold $(P=0.12)$ and Pedersen methods $(P=0.79)$. The mean neuroglycopenic symptom scores were similar between both groups. People with IAH according to Clarke, Gold and Pedersen declared a significantly greater poor hypoglycaemia symptoms score (Clarke: $P=4.46 \times 10^{-9}$, Gold: $P=6.73 \times 10^{-12}$, Pedersen: $\left.P=0.0006\right)$ as a cause for hypoglycaemia. The scores are detailed in Table 1.

\section{Patient Education}

Among the cohort, 51\% of people had completed a structured education programme and completion rates were similar between groups (Table 1). On the Clarke method, scores for knowledge to prevent future hypoglycaemic events were reduced in people with IAH compared to normal awareness $(P=0.04)$.

\section{Worry and Night-Time Hypoglycaemia}

Scores for night-time worry regarding hypoglycaemia $(P=0.04)$ and self-reported frequency of night hypoglycaemia $(P=0.001)$ were increased in people with IAH as compared to normal awareness, using the Clarke method. Scores for daytime worry regarding hypoglycaemia were similar in both groups (Table 1).

\section{Relationship Between Clarke, Gold and Pedersen Methods}

Spearman's correlation coefficients between Pedersen and Clarke and Pedersen and Gold 


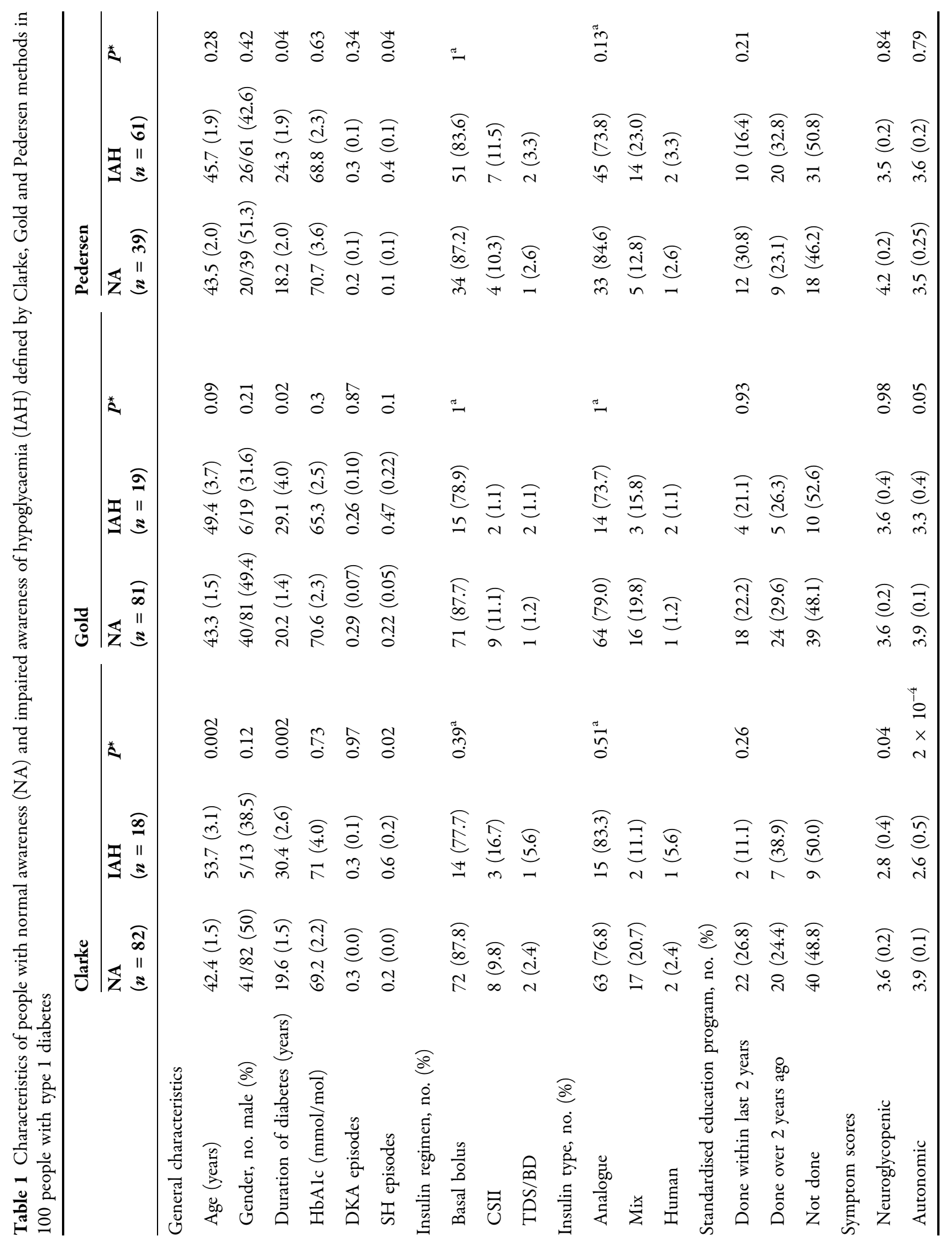




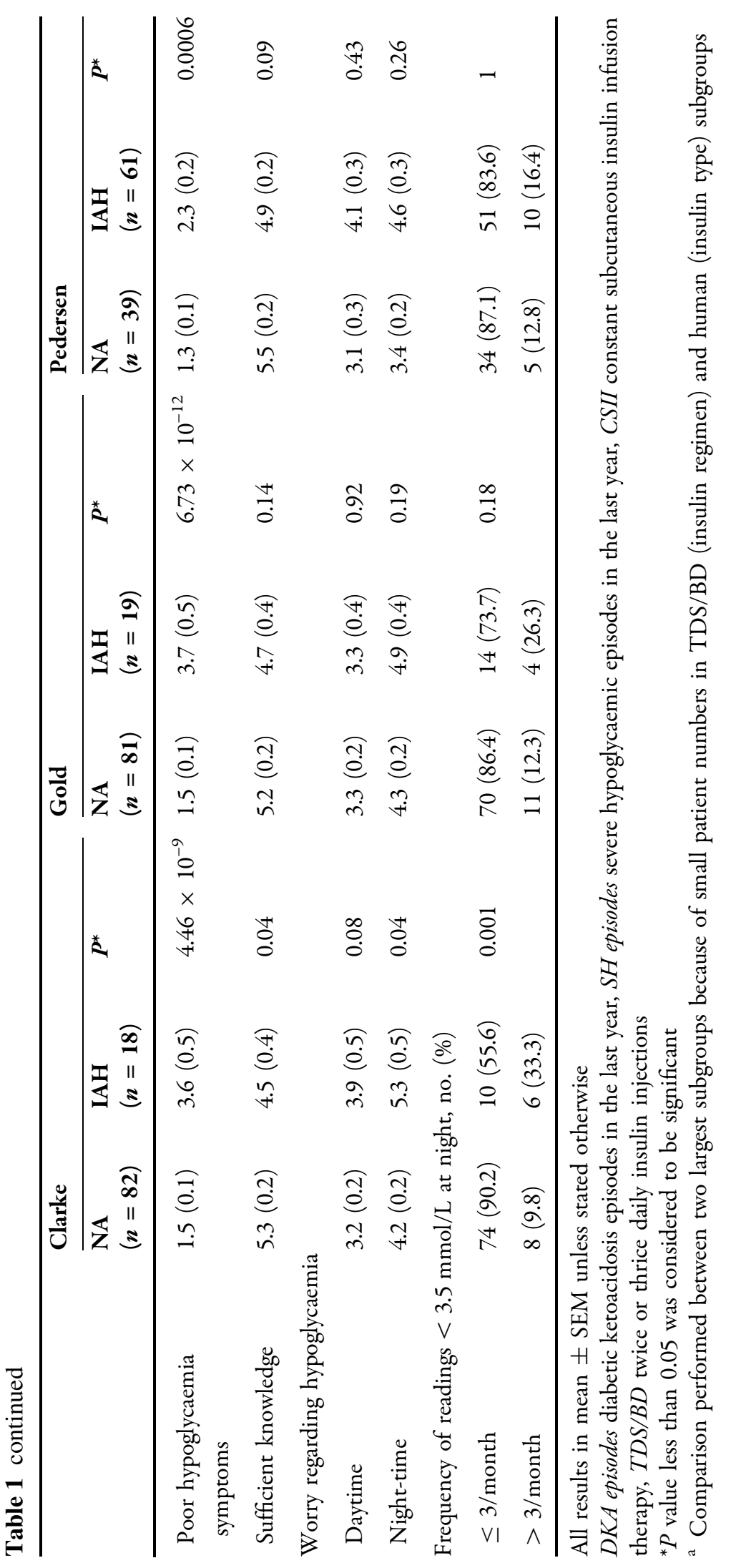


were $R_{\mathrm{s}}=0.555 \quad(P<0.001)$ and $R_{\mathrm{s}}=0.645$ $(P<0.001)$ respectively (Table 1$)$. The true positives, true negatives, false positives and false negatives of the Pedersen score based on the Clarke score were $17,44,38$ and 1 out of 100 patients respectively. The sensitivity, specificity, positive predictive value and negative predictive value of the Pedersen score based on the Clarke score was 94.4\%, 46.3\%, 27.9\% and 97.4\% respectively.

A moderate association between Clarke and Gold methods was observed $\left(R_{\mathrm{s}}=0.5669\right.$, $R^{2}=0.38, P<0.001$ ) (Fig. 1 ). The true positives, true negatives, false positives and false negatives of the Gold score based on the Clarke score were $11,74,8$ and 7 out of 100 patients respectively. The sensitivity, specificity, positive predictive value and negative predictive value of the Gold score based on the Clarke score was $61.1 \%$, $90.2 \%, 57.9 \%$ and $91.4 \%$ respectively.

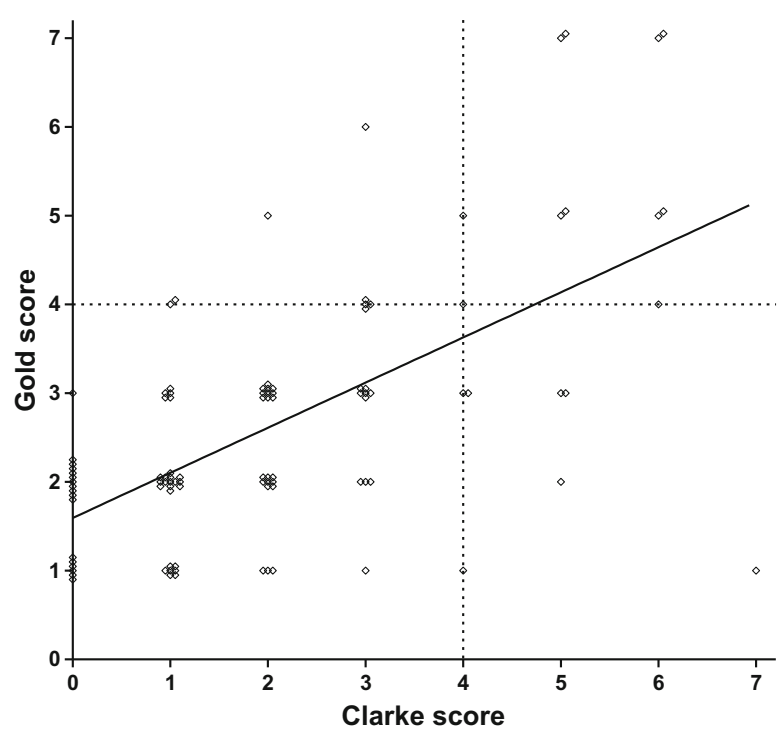

Fig. 1 Comparison of scores on Clarke and Gold methods. Each point on the scatter plot represents a single patient with respective Gold ( $y$-axis) and Clarke ( $x$-axis) scores. A line of best fit is drawn $\left(R_{\mathrm{s}}=0.5669, R^{2}=0.38\right.$, $p<0.01)$. On both Clarke and Gold methods, a score of 4 or above identifies IAH (dotted line)

\section{DISCUSSION}

This observational study in a large outpatient clinic population utilises and compares three validated methods to assess prevalence and characteristics of people with IAH $[9,15,16]$. The reported prevalence of IAH using all three methods is in line with recent estimates and is slightly reduced as compared to prevalence of approximately 25-30\% using Clarke and Gold methods in older reports [19-21]. A recent report suggests higher prevalence; however, that study enrolled people with type 1 diabetes with ongoing real-time CGM use who may have been recommended or met local funding criteria for real-time CGM on the basis of their awareness status [22]. The recent reduction in our study compared to older reports may reflect improvements in diabetes treatment, including standardised education. Previous evidence demonstrates that CSII reduces episodes of severe hypoglycaemia and structured education programmes, such as DAFNE, restore awareness of hypoglycaemia [12, 14]. In this study, no significant differences in treatment and education were noted between people with IAH and normal awareness; however, our study size was not powered to assess differences in this. Given the small number of patients who were excluded, it is unlikely that this has confounded the results. However, it is possible that exclusion of patients with advanced renal impairment from this study may have impacted on prevalence of IAH.

In accordance with previous reports, increased duration of diabetes, age (significant on Clarke method only) and severe hypoglycaemic episodes in the last year (significant on Clarke and Pedersen methods but not Gold method) were noted in people with IAH as compared with normal awareness [19]. Reduction in autonomic symptoms, one of the hallmarks of IAH, was significant in people with IAH compared to normal awareness, using the Clarke method, but not Gold or Pedersen methods [5]. This is in contrast to the previous reports suggesting increased age, severe hypoglycaemic episodes and reduced autonomic symptoms using the Gold method in a large 
study [20]. This difference is likely due to the decreased sensitivity of the Gold method compared to that of the Clarke method in defining IAH in smaller clinical settings.

The concordance of the three methods to identify IAH was evaluated and values were compared against each other. A previous study that evaluated the concordance between these three questionnaires in identifying people with IAH yielded controversial results $[19,23]$. In keeping with that previous report, the Pedersen method in this study was found to overestimate IAH and was a poor clinical discriminator of IAH and normal awareness [19]. However, in this study there was a moderate correlation between Clarke and Gold methods. This is in contrast to a previous report suggesting a strong correlation between both methods [19]. This study details the relationship between the two methods (Fig. 1). Although there is a correlation, our results suggest that this is modest with poor mutual concordance (Fig. 1).

The low sensitivity and positive predictive values of the Gold method against the Clarke method highlight the non-equivalence of these two questionnaires. This is also supported by different patient characteristics and features of IAH between Clarke and Gold methods, especially autonomic symptoms, suggesting that both methods are not equal. These differences may arise from different questionnaire designs. The Gold method relies upon a single-item response. This is more liable to bias, misinterpretation and measurement error as compared to multi-item response questionnaires, such as the Clarke method [24].

In this study we also demonstrate that IAH defined by the Clarke method is associated with decreased self-reported knowledge to avoid future hypoglycaemic events. Night-time worry and self-reported night-time hypoglycaemia are also increased in IAH people defined by the Clarke method. This highlights opportunities to improve education and behaviour in people with IAH, which may help reduce the incidence of hypoglycaemic episodes and improve longterm control. A stepped care approach of structured education and diabetes technologies, such as CSII and CGM to manage impaired awareness of hypoglycaemia and to reduce episodes of severe hypoglycaemia, has been previously recommended [25]. Fear and concerns regarding nocturnal hypoglycaemia in people with type 1 diabetes and families can substantially lead to behaviours such as overeating or insulin under-dosing which may impact on glycaemic control and diabetes complications [26].

One of the limitations of this study is that there is no gold standard test for IAH. Hypoglycaemia trials may benefit from access to patients with confirmed IAH on multiple modalities. Using such cases alongside hypoglycaemia-aware comparators in a prospective questionnaire study may provide the ability to construct more reliable ROC (receiver operating characteristic) analysis. A potential confounding factor that remains unresolved is the potential for demographics in altering the sensitivity of IAH assessment methods. The study was retrospective in nature, and large-scale prospective studies assessing the performance of each questionnaire in predicting the occurrence of severe hypoglycaemia are needed. Nevertheless, this study reveals important real-world insights regarding the utility of questionnairebased methods for assessing IAH.

\section{CONCLUSION}

This study reveals differences between characteristics of people with IAH defined by Clarke, Gold and Pedersen methods. Care must be taken when using these methods in clinical and research settings given their non-equivalence. The prevalence of IAH was overestimated using the Pedersen method for this cohort of people with type 1 diabetes, concordant with findings from a previous study [19]. The prevalence of IAH using the Clarke and Gold methods was $18 \%$ and $19 \%$, consistent with known evidence.

The National Institute for Health and Care Excellence (NICE) currently recommends the use of the Gold or Clarke scoring tools as methods to assess awareness of hypoglycaemia in people with type 1 diabetes [27]. Although there is no conclusive diagnostic investigation for IAH, our results suggest that care should be taken when using risk assessment scores and 
that multiple scoring modalities should be used in clinical settings to ensure validity of results with a reliable risk assessment. Further studies are needed to reassess the performance of questionnaire-based methods in larger populations and correlating them with metrics obtained from recent CGM systems with improved accuracies or prospective occurrence of severe hypoglycaemia episodes [22]. Future work focusing on CGM metrics and classification of hypoglycaemia awareness using functional brain imaging may provide us with more accurate diagnostic criteria for IAH.

\section{ACKNOWLEDGEMENTS}

The authors would like to thank the clinical team and participants of the study.

Funding. No funding or sponsorship was received for this study or the publication of this article.

Authorship. All named authors meet the International Committee of Medical Journal Editors (ICMJE) criteria for authorship for this article, take responsibility for the integrity of the work as a whole, and have given their approval for this version to be published.

Disclosures. Kshitiz Ghandi, Beatrice Pieri and Anne Dornhorst have nothing to disclose. Sufyan Hussain reports personal fees from Abbott Diabetes Care and Roche Diabetes care, outside the submitted work. The research work was performed at Imperial College London Healthcare NHS Trust. Writing of the manuscript was performed subsequently at King's College London. KG is currently at Health Education West Midlands, Birmingham, UK. Beatrice Pieri is currently at York Teaching Hospitals NHS Foundation Trust, York, UK. Sufyan Hussain is currently at Guy's and St Thomas' Hospital NHS Trust, London in addition to King's College London, London, UK.

Compliance with Ethics Guideline. The study was not required to have IRB approval as it used existing routine clinical data accessed directly by the clinical team caring for the patients and met local requirements to be undertaken as a clinical audit. No intervention was implemented on the patients for the purpose of the study, and no patient-identifiable information was used in the study. Retrospective analysis of questionnaires used in routine practice was approved as an audit by Imperial College Healthcare NHS Trust Clinical governance office. Informed consent was obtained from all participants at the time of questionnaire completion.

Data Availability. Anonymised datasets generated and/or analysed during the current study are available from the corresponding author on reasonable request from qualified researchers.

Open Access. This article is licensed under a Creative Commons Attribution-NonCommercial 4.0 International License, which permits any non-commercial use, sharing, adaptation, distribution and reproduction in any medium or format, as long as you give appropriate credit to the original author(s) and the source, provide a link to the Creative Commons licence, and indicate if changes were made. The images or other third party material in this article are included in the article's Creative Commons licence, unless indicated otherwise in a credit line to the material. If material is not included in the article's Creative Commons licence and your intended use is not permitted by statutory regulation or exceeds the permitted use, you will need to obtain permission directly from the copyright holder. To view a copy of this licence, visit http://creativecommons.org/licenses/bync/4.0/.

\section{REFERENCES}

1. Cryer P. Hypoglycaemia: The limiting factor in the glycaemic management of type I and type II diabetes. Diabetologia. 2002;45(7):937-48. 
2. Jacobson AM, Musen G, Ryan CM, et al. Long-term effect of diabetes and its treatment on cognitive function. N Engl J Med. 2007;356(18):1842-52.

3. Feltbower RG, Bodansky HJ, Patterson CC, et al. Acute complications and drug misuse are important causes of death for children and young adults with type 1 diabetes: results from the Yorkshire Register of diabetes in children and young adults. Diabetes Care. 2008;31(5):922-6.

4. Skrivarhaug T, Bangstad H-J, Stene LC, Sandvik L, Hanssen KF, Joner G. Long-term mortality in a nationwide cohort of childhood-onset type 1 diabetic patients in Norway. Diabetologia. 2006;49(2): 298-305.

5. Cryer P. Hypoglycemia in diabetes: pathophysiology, prevalence, and prevention. 3rd ed. Alexandria: American Diabetes Association; 2016.

6. Davis RE, Morrissey M, Peters JR, Wittrup-Jensen K, Kennedy-Martin T, Currie CJ. Impact of hypoglycaemia on quality of life and productivity in type 1 and type 2 diabetes. Curr Med Res Opin. 2005;21(9):1477-83.

7. Driver and Vehicle Licensing Agency. Diabetes mellitus: assessing fitness to drive-GOV.UK. 2016. https://www.gov.uk/guidance/diabetesmellitusassessing-fitness-to-drive. Accessed 18 Nov 2020.

8. Amiel SA. Hypoglycemia: from the laboratory to the clinic. Diabetes Care. 2009;32(8):1364-71.

9. Gold AE, MacLeod KM, Frier BM. Frequency of severe hypoglycemia in patients with type I diabetes with impaired awareness of hypoglycemia. Diabetes Care. 1994;17(7):697-703.

10. Cryer PE. Hypoglycemia begets hypoglycemia in IDDM. Diabetes. 1993;42(12):1691-3.

11. Choudhary P, Amiel SA. Hypoglycaemia: current management and controversies. Postgrad Med J. 2011;87(1026):298-306.

12. Hopkins D, Lawrence I, Mansell P, et al. Improved biomedical and psychological outcomes 1 year after structured education in flexible insulin therapy for people with type 1 diabetes: the U.K. DAFNE experience. Diabetes Care. 2012;35(8):1638-42.

13. van Beers CAJ, DeVries JH, Kleijer SJ, et al. Continuous glucose monitoring for patients with type 1 diabetes and impaired awareness of hypoglycaemia (IN CONTROL): a randomised, open-label, crossover trial. Lancet Diabetes Endocrinol. 2016;4(11): 893-902.

14. Pickup JC, Sutton AJ. Severe hypoglycaemia and glycaemic control in type 1 diabetes: meta-analysis of multiple daily insulin injections compared with continuous subcutaneous insulin infusion. Diabetes Med. 2008;25(7):765-74.

15. Clarke WL, Cox DJ, Gonder-Frederick LA, Julian D, Schlundt D, Polonsky W. Reduced awareness of hypoglycemia in adults with IDDM. A prospective study of hypoglycemic frequency and associated symptoms. Diabetes Care. 1995;18(4):517-22.

16. Pedersen-Bjergaard U, Agerholm-Larsen B, Pramming S, Hougaard P, Thorsteinsson B. Activity of angiotensin-converting enzyme and risk of severe hypoglycaemia in type 1 diabetes mellitus. Lancet. 2001;357(9264):1248-53.

17. Deary IJ, Hepburn DA, MacLeod KM, Frier BM. Partitioning the symptoms of hypoglycaemia using multi-sample confirmatory factor analysis. Diabetologia. 1993;36(8):771-7.

18. Pedersen-Bjergaard U, Pramming S, Thorsteinsson B. Recall of severe hypoglycaemia and self-estimated state of awareness in type 1 diabetes. Diabetes Metab Res Rev. 2003;19(3):232-40.

19. Geddes J, Wright RJ, Zammitt NN, Deary IJ, Frier BM. An evaluation of methods of assessing impaired awareness of hypoglycemia in type 1 diabetes. Diabetes Care. 2007;30(7):1868-70.

20. Geddes J, Schopman JE, Zammitt NN, Frier BM. Prevalence of impaired awareness of hypoglycaemia in adults with type 1 diabetes. Diabetes Med. 2008;25(4):501-4.

21. Choudhary P, Geddes J, Freeman JV, Emery CJ, Heller SR, Frier BM. Frequency of biochemical hypoglycaemia in adults with type 1 diabetes with and without impaired awareness of hypoglycaemia: no identifiable differences using continuous glucose monitoring. Diabetes Med. 2010;27(6):666-72.

22. Lin $Y$, Hung M, Sharma A, et al. Impaired awareness of hypoglycemia continues to be a risk factor for severe hypoglycemia despite the use of continous glucose monitors in type 1 diabetes. Endocr Pract. 2019;25(6):517-25.

23. Pedersen-Bjergaard U, Høi-Hansen T, Thorsteinsson B. An evaluation of methods of assessing impaired awareness of hypoglycemia in type 1 diabetes: response to Gedded et al. Diabetes Care. 2007;30(11):e112.

24. Rattray J, Jones MC. Essential elements of questionnaire design and development. J Clin Nurs. 2007;16(2):234-43.

25. Yeoh E, Choudhary P, Nwokolo M, Ayis S, Amiel SA. Interventions that restore awareness of hypoglycemia in adults with type 1 diabetes: a 
systematic review and meta-analysis. Diabetes Care. 2015;38(8):1592-609.

26. Amiel SA. Hypoglycemia at night: a cause for alarm? Diabetes Technol Ther. 2012;14(2):99-100.
27. National Institute for Health and Care Excellence. Type 1 diabetes in adults: diagnosis and management | Guidance NICE. NICE. 2015.https://www. nice.org.uk/guidance/ng17. Accessed 18 Nov 2020. 